\title{
Dependence of Galaxy Shape on Environment in the Sloan Digital Sky Survey
}

\author{
Frederick Kuehn \\ Department of Physics, The Ohio State University, Columbus, OH 43210 \\ kuehn@mps.ohio-state.edu \\ and \\ Barbara S. Ryden \\ Department of Astronomy, The Ohio State University, Columbus, OH 43210 \\ ryden@astronomy. ohio-state.edu
}

\begin{abstract}
Using a sample of galaxies from the Sloan Digital Sky Survey Data (SDSS) Data Release 4, we study the trends relating surface brightness profile type and apparent axis ratio to the local galaxy environment. We use the SDSS parameter 'fracDeV' to quantify the profile type (fracDeV $=1$ for a pure de Vaucouleurs profile; fracDeV $=0$ for a pure exponential profile). We find that galaxies with $M_{r} \gtrsim-18$ are mostly described by exponential profiles in all environments. Galaxies with $-21 \lesssim M_{r} \lesssim-18$ mainly have exponential profiles in low density environments and de Vaucouleurs profiles in high density environments. The most luminous galaxies, with $M_{r} \lesssim-21$, are mostly described by de Vaucouleurs profiles in all environments. For galaxies with $M_{r} \lesssim-19$, the fraction of de Vaucouleurs galaxies is a monotonically increasing function of local density, while the fraction of exponential galaxies is monotonically decreasing. For a fixed surface brightness profile type, apparent axis ratio is frequently correlated with environment. As the local density of galaxies increases, we find that for $M_{r} \in[-18,-20]$, galaxies of all profile types become slightly rounder, on average; for $M_{r} \in[-20,-22]$, galaxies with mostly exponential profiles tend to become flatter, while galaxies with de Vaucouleurs profiles tend to become rounder; for $M_{r} \in[-22,-\infty]$, galaxies with mostly exponential profiles become flatter, while the de Vaucouleurs galaxies become rounder in their inner regions, yet exhibit no change in their outer regions. We comment on how the observed trends relate to the merger history of galaxies.
\end{abstract}

Subject headings: galaxies: elliptical and lenticular, cD — galaxies: fundamental parameters — galaxies: photometry — galaxies: spiral — galaxies: statistics 


\section{INTRODUCTION}

The modern paradigm of hierarchical galaxy formation is rooted in the notion that large stellar systems are formed through the mergers of smaller ones. Understanding the creation and evolution of a particular galaxy requires a knowledge of the merger history of dark halos and the accompanying gas dynamics. Different environments necessarily lead to differing merger histories, resulting in differences in the observable properties of each galaxy. In particular, denser environments lead to more frequent mergers, which cause significant structural changes to galaxies. Also, gravitational harassment among galaxies in dense regions can affect the morphological properties of galaxies. Within the context of the current paradigm of galaxy formation, it is possible to create computer simulations that begin with some matter distribution and then follow the creation and evolution of structure (e.g. see Bertschinger (1998); Abadi et al. (2003); Bell et al. (2005); Robertson et al. (2005)). Observational astronomy, by contrast, is unable to follow the evolution of a particular galaxy. However, statistical correlations between properties such as galaxy color, luminosity, kinematics, and shape have been found. Attempts to understand how such trends are related to the underlying physics of galaxy evolution are a major focus of extragalactic astronomy. The purpose of this study is to examine both new and well known trends that relate a galaxy's structure to its environment through the use of observable quantities.

Historically, Hubble (1926) classified galaxies by their visual appearance on photographic plates. Elliptical galaxies have smooth elliptical isophotes; spiral galaxies have spiral arms that wind outward from a central bulge or bar. By measuring the surface brightness $I(R)$ along the major axis of a galaxy's image, it was noticed that bright $\left(M_{B} \lesssim-20\right)$ ellipticals have light profiles that are well fit by $\log I \propto-\mathrm{R}^{1 / 4}$ (de Vaucouleurs 1959). On the other hand, the azimuthally averaged surface brightness profiles of spiral galaxies were discovered to have $\log I \propto-\mathrm{R}$ (Freeman 1970). Such observations reveal a correlation of surface brightness profile with Hubble type. Furthermore, elliptical galaxies are slowly rotating systems characterized by triaxial shapes; in contrast, spiral galaxies are rapidly rotating flattened structures. It has also been found that the average color of galaxies differs for the different galaxy types; ellipticals tend to be redder, while spirals are bluer. Such correlations with Hubble type exist for a host of galaxy properties.

There are well known trends relating morphological type to the local density of galaxies (Hubble \& Humason 1931; Dressler 1980; Goto et al. 2003; Tanaka et al. 2004; Postman et al. 2005). In general, bright elliptical galaxies are preferentially found in regions of higher galaxy number density; on the other hand, spirals account for the majority of bright galaxies in the field. It is worth noting, however, that such studies have been focused on galaxies with $M_{r} \lesssim-20$. New, large galaxy surveys open up the possibility of exploring such trends in different magnitude bands.

Images of galaxies are a projection of the true shape. Most studies of the axis ratio of galaxies have concentrated on understanding what the distribution of apparent axis ratios tells us about the intrinsic axis ratios (e.g. Sandage et al. (1970); Binney \& de Vaucouleurs (1981); Lambas et al. (1992); Elmegreen et al. (2004); Vincent \& Ryden (2005)). Such information is a necessary 
ingredient for, among other things, understanding the kinematic properties of galaxies. Generally, the distribution of apparent axis ratios for thin, nearly circular disks is an approximately uniform function, giving an average axis ratio of $\sim 0.5$. For elliptical galaxies, which tend to be triaxial (Lambas et al. 1992; Vincent \& Ryden 2005), the distribution of apparent axis ratios is a steeply peaked function with an average value of $\sim 0.7$. It is also found that the average axis ratio for ellipticals depends on luminosity, with brighter ellipticals being rounder (Vincent \& Ryden 2005). As previously mentioned, bright ellipticals tend to be found in high density environments. This suggests that the eventful merger histories of these systems lead to systematic change in average axis ratio. One under-studied area, related to galaxy shape, is the dependence of axis ratio on environment. Lambas et al. (1992) briefly comment on this dependence for elliptical galaxies. However, a continued investigation of this field will help complete understanding of the kinematical properties of galaxies.

In this paper, we use photometric data from the Sloan Digital Sky Survey Data Release 4 (SDSS DR4) (Adelman-McCarthy et al. 2005) to study the correlations of two structural properties of galaxies with environment; namely, surface brightness profile and apparent axis ratio. The SDSS provides an unprecedentedly large data set with a uniform data reduction scheme, allowing for a consistent study. SDSS data has already been used to quantify correlations between many galaxy properties (Blanton et al. 2003a; Goto et al. 2003; Hogg et al. 2003; Blanton et al. 2004; Hogg et al. 2004; Tanaka et al. 2004).

In $\S 2$ of this paper, we describe the SDSS, our morphological classification scheme, and the methods by which we determine the apparent axis ratio of galaxies. In $\S 3$, we present our results on the surface brightness profile type - environment relationship. We make two extensions to previous studies. First, we use galaxies in the relatively broad absolute magnitude range $M_{r} \in[-17,-23]$. Second, we subdivide the data into narrow magnitude bins to determine the luminosity dependence of the profile type - environment relation. In $\S 4$, we describe the results of our study of the apparent axis ratio - environment correlation. Motivation for this research was initiated by a prior analysis of galaxy axis ratios in the SDSS data (Vincent \& Ryden 2005), where it was proposed that axis ratio could be correlated with environment. In $\S 5$ we place our results in the context of the current understanding of galaxy formation and evolution.

\section{DATA}

The Sloan Digital Sky Survey (York et al. 2000; Stoughton, et al. 2002) will, when complete, provide a map of nearly one-fourth of the celestial sphere. A CCD mosaic camera (Gunn et al. 1998) images the sky in five photometric bands (ugriz; Fukugita et al. (1996); Smith et al. (2002)). The follow-up spectroscopic survey provides spectra for galaxies to a limiting Petrosian magnitude $m_{r}=17.77$. The Sloan Digital Sky Survey Data Release 4, released to the astronomical community in 2004 October, covers a photometric area of $6670 \mathrm{deg}^{2}$ and a spectroscopic area of $4783 \mathrm{deg}^{2}$ (Adelman-McCarthy et al. (2005); see also Stoughton, et al. (2002), Abazajian et al. 
(2003), Abazajian et al. (2004), and Abazajian et al. (2005)).

The SDSS DR4 data processing pipeline provides a morphological star/galaxy separation, with extended objects being classified as 'galaxies' and unresolved objects being classified as 'stars'. For each galaxy, in each photometric band, two models are fitted to the two-dimensional galaxy image. The first model has a de Vaucouleurs surface profile (de Vaucouleurs 1948):

$$
I(R)=I_{e} \exp \left(-7.67\left[\left(R / R_{e}\right)^{1 / 4}-1\right]\right),
$$

which is truncated beyond $7 R_{e}$ to go smoothly to zero at $8 R_{e}$. The second model has an exponential profile:

$$
I(R)=I_{e} \exp \left(-1.68\left[R / R_{e}-1\right]\right),
$$

which is truncated beyond $3 R_{e}$ to go smoothly to zero at $4 R_{e}$. For each model, the apparent axis ratio $q_{m}$ and the phase angle $\varphi_{m}$ are assumed to be constant with radius. The parameters $q_{m}$, $\varphi_{m}, R_{e}$, and $I_{e}$ are varied to give the best $\chi^{2}$ fit to the galaxy image, after convolution with a double-Gaussian fit to the point spread function.

A further fit to each galaxy is made by taking the best de Vaucouleurs model and the best exponential model, and finding the linear combination of the two that gives a new best fit. The fraction of the total flux contributed by the de Vaucouleurs component is the parameter fracDeV, which is constrained to lie in the interval $[0,1]$. The fracDeV parameter is functionally equivalent to the Sérsic (1968) index $n$ in the interval $1 \leq n \leq 4$ (Vincent \& Ryden 2005). If a galaxy has a Sérsic index $n$, then $n=1$ corresponds to fracDeV $=0, n=4$ corresponds to fracDeV $=1$, and the dependence of fracDeV upon $n$ is monotonic in the interval $1 \leq n \leq 4$. Following Vincent \& Ryden (2005), we call galaxies with $0 \leq$ fracDeV $\leq 0.1(n \lesssim 1.2)$ ex galaxies. Galaxies with $0.1 \leq$ fracDeV $\leq 0.5(1.2 \lesssim n \lesssim 2.0)$ are labeled $e x /$ de galaxies. Galaxies with $0.5 \leq$ fracDeV $\leq 0.9$ $(2.0 \lesssim n \lesssim 3.3)$ are labeled $d e / e x$ galaxies. Finally, galaxies with fracDeV $\geq 0.9(n \gtrsim 3.3)$ are labeled de galaxies.

The SDSS DR4 databases provide many different measures of the apparent axis ratio $q$ of each galaxy in each of the five photometric bands. In this paper, we use the $r$ band data, at an effective wavelength of $6165 \AA$; this is the band in which the star/galaxy classification is made. A useful measure of the apparent shape in the outer regions of galaxies is the axis ratio of the 25 mag $\operatorname{arcsec}^{-2}$ isophote. The SDSS DR4 data pipeline finds the best fitting ellipse to the 25 mag $\operatorname{arcsec}^{-2}$ isophote in each band; the semimajor axis and semiminor axis of this isophotal ellipse are $A_{25}$ and $B_{25}$. The isophotal axis ratio $q_{25} \equiv B_{25} / A_{25}$ then provides a measure of the apparent galaxy shape at a few times the effective radius. For galaxies in our sample with fracDeV $=1, A_{25} \sim 3.2 R_{e}$; for galaxies with fracDeV $=0, A_{25} \sim 2.4 R_{e}$.

The second measure of the apparent axis ratio that we use is $q_{\mathrm{am}}$, the axis ratio determined by the use of adaptive moments of the galaxy's surface brightness. The method of adaptive moments determines the $n$th order moments of a galaxy image, using an elliptical weight function whose shape matches that of the image (Bernstein \& Jarvis 2002; Hirata \& Seljak 2003). The SDSS 
DR4 adaptive moments use a weight function $w(x, y)$ that is a Gaussian matched to the size and ellipticity of the galaxy image $I(x, y)$. The adaptive first order moments,

$$
\vec{x}_{0}=\frac{\int \vec{x} w(x, y) I(x, y) d x d y}{\int w(x, y) I(x, y) d x d y},
$$

tell us the 'center of light' of the galaxy's image. With this knowledge, we can compute the adaptive second order moments:

$$
M_{x x}=\frac{\int\left(x-x_{0}\right)^{2} w(x, y) I(x, y) d x d y}{\int w(x, y) I(x, y) d x d y},
$$

and so forth. The SDSS DR4 provides for each image the values of the parameters $\tau=M_{x x}+M_{y y}$, $e_{+}=\left(M_{x x}-M_{y y}\right) / \tau$, and $e_{x}=2 M_{x y} / \tau$. The adaptive second moments can be converted into an axis ratio using the relation

$$
q_{\mathrm{am}}=\left(\frac{1-e}{1+e}\right)^{1 / 2},
$$

where $e=\left(e_{+}^{2}+e_{\times}^{2}\right)^{1 / 2}$. The adaptive moments axis ratio $q_{\mathrm{am}}$ is not corrected for the effects of seeing. The SDSS DR4 also provides the fourth order adaptive moments of the galaxy image, and the adaptive moments $\tau_{\mathrm{psf}}, e_{+, \mathrm{psf}}$, and $e_{\times, \mathrm{psf}}$ of the point spread function at the galaxy's location. These moments can be used to correct for the smearing and shearing due to seeing; such corrections are essential for studying the small shape changes resulting from weak lensing (Bernstein \& Jarvis 2002; Hirata \& Seljak 2003). However, when we examine the apparent axis ratios of galaxies in Section 4 , we will only look at well-resolved galaxies $\left(\tau>6.25 \tau_{\mathrm{psf}}\right)$, for which the seeing corrections are negligible. We note that, at most, weak lensing shears the shape of a galaxy at the one percent level (Sheldon et al. 2004). Such minute changes in the shape do not significantly affect our results.

Our complete sample of galaxies consists of those objects in the SDSS DR4 spectroscopic sample which are flagged as galaxies, which have $\tau \geq \tau_{\text {psf }}$, and which have spectroscopic redshifts $z>0.004$ and a redshift confidence parameter $z$ conf $>0.35$. The absolute magnitude $M_{r}$ of each galaxy is computed from its SDSS cmodel apparent magnitude, assuming a uniform Hubble flow with $H_{0}=70 \mathrm{~km} \mathrm{~s}^{-1} \mathrm{Mpc}^{-1}, \Omega_{M, 0}=0.3$, and $\Omega_{\Lambda, 0}=0.7$. The complete sample contains $N=305558$ galaxies with a median redshift of $z \sim 0.1$. The morphology of each galaxy in the sample is quantified by the parameter fracDeV, and by the two measures of the apparent axis ratio: $q_{25}$, which gives the shape in the outer region, and $q_{\mathrm{am}}$, which is more strongly weighted toward the inner region of the galaxy.

\section{PROFILE TYPE}

\subsection{SURVEY VOLUME}

A galaxy with absolute magnitude $M_{r}$ will enter the SDSS spectroscopic survey if its luminosity distance is less than $d_{L}=10^{0.2\left(m_{r}-M_{r}-25\right)} \mathrm{Mpc}$, where the limiting apparent magnitude of the 
survey is $m_{r}=17.77$. In our study of the relationship between surface brightness profile type and environment, we create six volume-limited samples with absolute magnitude cut-offs $M_{r_{0}}=-17$, $-18,-19,-20,-21$, and -22 . The maximum luminosity distance in the volume-limited samples ranges from $d_{L}=10^{1.954} \mathrm{Mpc}=90 \mathrm{Mpc}$ for the $M_{r_{0}}=-17$ sample (corresponding to $z_{\max } \approx 0.02$ ), to $d_{L}=10^{2.954} \mathrm{Mpc}=900 \mathrm{Mpc}$, for the $M_{r_{0}}=-22$ sample (corresponding to $z_{\max } \approx 0.2$ ). In each sample we choose as our 'target galaxies' those galaxies with $M_{r} \in\left[M_{r_{0}}, M_{r_{0}}-1\right]$. The target galaxies are those galaxies for which we will determine the profile type - environment dependence. The total number of target galaxies in each volume-limited sample, and the fraction of target galaxies corresponding to each of our four morphological types, is presented in Table 1. Table 1 also lists, under the heading 'cylinder width', the total number of cylinders (see §3.2) that do not intersect the border of the SDSS spectrographic survey, which is the same as the number of galaxies that we keep in our analysis (see §3.2). The galaxies in each sample are subject to the quality cuts described in $\S 2$.

\subsection{THE ENVIRONMENT}

The environment of each target galaxy is determined by a count of neighboring galaxies. We adhere to the following in determining this count. A cylinder is created around each target galaxy in redshift space, with the long axis of the cylinder lying along the line of sight. The cylinder stretches $6 h^{-1} \mathrm{Mpc}$ (with $h=0.7$ ) in either radial direction from the target galaxy (i.e. $12 h^{-1}$ $\mathrm{Mpc}$ in total). The radius of the cylinder is one of the following: $0.5 h^{-1} \mathrm{Mpc}, 2 h^{-1} \mathrm{Mpc}, 8$ $h^{-1} \mathrm{Mpc}$. Counting the number of galaxies with $M_{r} \leq M_{r_{0}}$ in this cylinder defines our density parameter. We note that in dense environments, this method undercounts the true number of galaxies in each cylinder. This is because the spectroscopic fibers on each SDSS plate cannot be placed more closely than $55^{\prime \prime}$. This causes $\sim 7 \%$ of galaxies (of the total) to be lost (Blanton et al. 2003a), undercounting galaxies in high density regions. Fibers are assigned to galaxies with no correlations to galaxy properties (Blanton et al. 2003b). Hence, there should be no significant effect to our results. For example, if $7 \%$ of each galaxy type in the highest density bin were moved to the second-highest density bin, the relative fraction would not change. In general, this is the largest source of galaxy loss, as the completeness of the spectroscopic survey is $\sim 91 \%$ (Blanton et al. 2003a).

The SDSS takes spectra using circular plates whose diameter subtends an angle of $3^{\circ}$ on the sky. The SDSS DR4 spectroscopic survey does not completely match the area covered by the DR4 photometric survey. Figure 1 shows the boundary of the spectroscopic survey. If a target galaxy is within one cylinder width of the border, it is dismissed from our analysis. In addition, if a galaxy is within one-half cylinder length, $6 h^{-1} \mathrm{Mpc}$, of the maximum luminosity distance, it is dismissed from our analysis.

In determining the environment for target galaxies in each magnitude bin we count all of the galaxies brighter than the magnitude cut-off $M_{r_{0}}$. In plotting our results, we group together at least 
100 galaxies in each bin. We take the weighted mean of the number of galaxies in each cylinder that falls into a particular bin. This defines the local density, or environment for all galaxies in each bin. The highest density bin may have as few as 50 galaxies (the only excpetion to this rule is the case with $M_{r_{0}}=-17$ and $2 h^{-1}$ Mpc cylinders where the number in each bin is half that just described). We use the same density bins for all profile types so that we may determine the relative fraction of each. The bins are determined by the binning of the ex galaxies subject to the previously given conditions. We changed the possible binning for this analysis, varying it by profile type, and found no substantial change in the results.

\subsection{RESULTS FOR PROFILE TYPE - ENVIRONMENT RELATIONSHIP}

Many studies (e.g. Dressler (1980); Goto et al. (2003); Postman et al. (2005)) have shown that as the density of galaxies in a region increases, the relative fraction of elliptical galaxies to spiral galaxies increases. Instead of using the Hubble classification scheme, we classify galaxies based on their surface brightness profile type, using four classes ranging from the highly concentrated de galaxies to the least concentrated $e x$ galaxies.

Our largest survey volume extends to the distance at which a galaxy with $M_{r}=-22$ has $m_{r}=17.77$, corresponding to $z \approx 0.2$. Nuijten et al. (2005) have shown that in the redshift interval $0<z<0.2$, there is little evolution in the fraction of galaxies with $n>2$ (corresponding to our classes de and de/ex). Since all our other magnitude cut-offs correspond to lower maximum redshifts, we do not expect significant redshift evolution effects in our samples.

Figures 2 through 4 display our findings on the trends of galaxy profile with environment. The plots are organized by magnitude cut-off and cylinder radius. The ex galaxies are represented by green solid lines and solid circles; the $e x / d e$ galaxies, by blue dotted lines and solid triangles; the de/ex galaxies, by red dashed lines and open circles; the de galaxies, by magenta long-dashed lines and open triangles. The plotted errors are the expected errors in the mean, assuming Poisson statistics. Table 1 lists the number of galaxies in each of our volume-limited surveys. It also lists the fraction of each profile type. Finally, it lists the total number of galaxies that survive all of our quality cuts (see $\S 2$ and $\S 3.2$ ).

We summarize the results as follows:

1. Figure 2 displays the results for cylinders of width $0.5 h^{-1} \mathrm{Mpc}$. There are four primary results. First, galaxies with $M_{r} \gtrsim-19$ are found to have mostly exponential light profiles in all environments. Second, galaxies with $M_{r} \lesssim-21$ have mostly de Vaucouleurs light profiles in all environments. Third, for galaxies with $M_{r} \lesssim-19$, as local density increases, the fraction of de Vaucouleurs galaxies increases monotonically with increasing local density, while the fraction of exponentials decreases. Fourth, there is a crossover of predominant profile type between high and low density environments only for galaxies with $M_{r} \sim-20$. Plotting results for so many magnitude bins clearly shows how the change in dominant profile type depends not only on environment, but 
also on absolute magnitude. Dressler (1980) examined the morphology - environment relation for galaxies with $M_{V}<-19.67$ (assuming $h=0.7$ ); for a typical color $B-V \approx 0.9$, this corresponds roughly to $M_{r} \lesssim-20$.

2. Figures 3 and 4 display the effect of increasing the cylinder size. The main result is that trends are washed out on scales larger than about $2 h^{-1}$ Mpc. There is no plot for the magnitude cut-offs $-17,-18$, and -19 for cylinders that are $8 h^{-1} \mathrm{Mpc}$ wide; the volume of space probed is small enough that once all of the cylinders that intersect with the border are thrown out, there are too few galaxies left over to make the plot statistically interesting.

\section{APPARENT AXIS RATIO}

This section of the paper addresses how the average shape of galaxies, as quantified by their apparent axis ratio, depends on their environment, for a fixed luminosity and profile type. Our analysis of this topic is described below. We first highlight any differences in the methodology from the preceding section. Unless otherwise mentioned, our methods here remain the same as those described in $\S 3$.

One idiosyncrasy discovered in Vincent \& Ryden (2005) is that for fixed luminosity the average axis ratio is not always a monotonic function of Sérsic index. Subdividing all galaxies into only two groups, one with all galaxies with fracDeV $<0.5$, or $n \lesssim 2$, and another with the remaining galaxies, would average over this interesting behavior. This provides us with further motivation for maintaining our galaxy classification scheme, where we place galaxies in one of the four groups: ex, $e x / d e, d e / e x$ and $d e($ see $\S 2)$.

\subsection{SURVEY VOLUME}

The survey volume for this analysis is similar to that described in $§ 3.1$. An important difference is that we place a stricter cut on the point spread function (see §2) and in the process lose many de Vaucouleurs galaxies. In Vincent \& Ryden (2005) it was noted that there is a luminosity axis ratio trend. In the range $[-18,-20]$ the trend is approximately flat. In the range $[-20,-22]$ the axis ratio increases with increasing luminosity. For galaxies brighter than -22 , the axis ratio seems to level off. In principle, this correlation could affect our results. This motivates us to keep luminosity bins as narrow as possible, so as to minimize any effect of this trend within a single bin. On the other hand, statistical claims are only useful for large sample sizes. To keep our subsamples of galaxies large enough, we are motivated to keep luminosity bins as wide as possible. As a compromise, we make luminosity bins two magnitudes wide.

We start by creating three volume-limited surveys with absolute magnitude cut-offs $M_{r_{0}}=$ $-18,-20$, and -22 . For the volumes corresponding to the first two cut-offs, we determine the 
environment (see §3.2) for target galaxies that have absolute magnitude $M_{r} \in\left[M_{r_{0}}, M_{r_{0}}-2\right]$, while for the brightest cut-off we find the environment for all galaxies with $M_{r} \in[-22,-\infty]$. There are only sixteen galaxies that are brighter than -24 in absolute magnitude in our volume; therefore, their inclusion does not significantly widen the effective magnitude range of the bin. For example, in the volume corresponding to the $M_{r_{0}}=-18$ cut-off we find the environment for all galaxies with $M_{r} \in[-18,-20]$ where the luminosity distance of a galaxy is no larger than $d_{L}=143 \mathrm{Mpc}$, corresponding to the maximum luminosity distance at which a galaxy of absolute magnitude $M_{r_{0}}=-18$ could be observed. The total number of galaxies in each volume, and the percentage of each profile type is presented in Table 2. Table 2 also lists, under the heading 'cylinder width', the total number of cylinders (see §3.2) that do not intersect the border of the SDSS spectrographic survey, which is the same as the number of target galaxies that we keep in our analysis. The galaxies in each sample are subject to quality cuts which are described in $\S 2$. The binning procedure is described in $§ 3.2$.

\subsection{RESULTS FOR THE APPARENT AXIS RATIO - ENVIRONMENT RELATIONSHIP}

Figures 5 through 7 show the correlations of apparent axis ratio with environment, labeled by profile type. Tables 3 through 5 contain all related statistical information. We plot a best fit straight line through the binned data that minimizes the chi-square. The value of the reduced chi-square for each fit is printed in the tables. The error bars show the error in the mean assuming Poisson statistics. The choice of colors, line type and point type are the same as previously described in $\S 3.3$.

Since the average axis ratio depends on the average luminosity, the normalization of each best fit line, for the axis ratio - environment trend, is set by the average luminosity for that profile type. Also, since the luminosity functions for each profile type are different in the different volumelimited samples, the average luminosity of each profile type is slightly different. For example, in the $M_{r} \in[-20,-22]$ range, the $e x$ galaxies have an average magnitude of $M_{r} \approx-20.5$, while the de galaxies have $M_{r} \approx-21.3$. From the luminosity - axis ratio trend, it is expected that the de galaxies would be rounder. This is reflected in Figure 6, say, where the trend for the de galaxies lies above the trend for the ex galaxies. Therefore, while it is useful to compare the difference in the axis ratio as determined by the $25 \mathrm{mag} \operatorname{arcsec}^{-2}$ isophote versus the axis ratio as determined by the adaptive moments technique, comparisons of axis ratios between galaxy profile types need to be made with the caveat that they contain different luminosity information.

In the absolute $r$ band magnitude range $[-20,-22]$, it has been shown (Vincent \& Ryden 2005) that, for all profile types, there is a trend toward roundness as galaxies become brighter. In particular, the average axis ratio may increase by as much as approximately 0.05 when the magnitude decreases by unity (Vincent \& Ryden 2005). However, we find that the luminosity in

each local density bin is equal to within one tenth of a magnitude confirming that any trend we 
see are not due to luminosity. Specifically, for $M_{r} \in[-20,-22]$, the average magnitude for the $e x$ galaxies is -20.5 , for the $e x / d e$ galaxies it is -20.7 , for the $d e / e x$ galaxies it is -21.0 and for the de galaxies it is -21.3 . In other magnitude ranges trends of mean axis ratio with luminosity are weaker and we also find that magnitude does not vary significantly from bin to bin.

Table 2 lists the number of galaxies in each of our volume-limited samples. Also, it lists the fraction of galaxies of each profile type. Tables 3 through 5 list all statistical information related to each profile type and cylinder size: the chi-square of the best fit line; whether the axis ratio is increasing ('+') or decreasing ('-') with increasing local density; our statistic labeled 'significance' is the ratio of the slope to its standard deviation, characterizing if the slope is significantly different than zero.

We summarize the results.

1. In the magnitude range $[-18,-20]$ the data show one statistically important trend at the $3 \sigma$ level. The $e x / d e$ galaxies get rounder as the local density of galaxies increases, and the axis ratio, as measured by the axis ratio determined by the moments, for the de/ex galaxies increases with density. Other profile types in this magnitude range tend to become rounder at higher local density, but at a lower level of statistical significance. Plots are only created for cylinders of width $0.5 h^{-1} \mathrm{Mpc}$ and $2 h^{-1} \mathrm{Mpc}$. When cylinders of width $8 h^{-1} \mathrm{Mpc}$ are considered, too many are near the border leaving a statistically uninteresting sample.

2. In the magnitude range $[-20,-22]$ we find the strongest trends between axis ratio and environment. Generally, the $d e$ and $d e / e x$ galaxies get rounder with increasing local density. This trend is more pronounced in the inner regions of these galaxies. On the other hand, the $e x / d e$ galaxies tend toward greater flattening as the local density of galaxies increases, with a similar trend, though less statistically significant, observed for the ex galaxies. Trends of axis ratio with environment are only significant on scales less than or equal to $2 h^{-1} \mathrm{Mpc}$.

3. For our sample of the brightest galaxies, in the magnitude range $[-22,-\infty]$, we find that trends are more pronounced in the inner regions than the outer regions; trends in the shape, determined by the moments of the light distribution, are no longer present when we use the axis ratio determined by the $25 \mathrm{mag} \operatorname{arcsec}^{-2}$ isophote. This is especially true of the de galaxies. Statistically, they show a strong positive trend in their axis ratio as determined by the moments of their light distribution. However, the average axis ratio for these galaxies derived from the $25 \mathrm{mag}$ $\operatorname{arcsec}^{-2}$ isophote is essentially constant with environment.

\section{DISCUSSION}

The novel aspect of our study of the surface brightness profile type - environment relationship is our use of a large magnitude range subsequently divided into many volume-limited samples. Averaging over a wide magnitude range blurs out some of the observed trends, resulting in an 
inability to distinguish them. Using many volume-limited samples allows us to determine that, at absolute $r$ band magnitudes around -17 , galaxies are almost all described by purely exponential light profiles at all densities, with no strong trends in the fraction of different profile types with environment. For absolute magnitudes around -19 and -20 there is a crossover from low densities to high densities; galaxies with exponential profiles are more common at low densities, while at high local densities galaxies are most likely to be well described by a de Vaucouleurs profile. Finally, for bright galaxies with $M_{r} \sim-22$, most galaxies have de Vaucouleurs profiles.

These results fit well into the hierarchical structure formation picture. Generally, the result of mergers is to increase entropy. In spiral galaxies, stellar orbits in the disk are nearly circular and all of the disk stars travel in the same direction, corresponding to a low overall entropy. On the other hand, orbits of stars in elliptical galaxies are unsystematic, giving rise to a high entropy system. Moreover, each of these two systems have different light profiles. Spiral galaxies tend to be less concentrated and have exponential profiles; ellipticals have more of their light concentrated near their center and most bright ellipticals have de Vaucouleurs profiles. We find that the dimmer galaxies, which have presumably experienced fewer mergers, are mostly well described by exponential profiles. For galaxies in the middle of our luminosity range, the environment is strongly correlated with profile type. Galaxies in high density environments experience, on average, more mergers than those in low density environments. The brightest galaxies, and presumably the ones that have been created through many mergers, are predominantly de Vaucouleurs.

The dependences of profile type and apparent axis ratio on environment show statistically interesting trends on scales up to $2 h^{-1} \mathrm{Mpc}$. This is consistent with other studies of the correlations of galactic properties with environment (Blanton et al. 2004). The properties of a galaxy are generally affected by the presence of other galaxies in the same group or cluster, but not by galaxies in neighboring clusters.

Lambas et al. (1992) use the APM Bright Galaxy Survey (Maddox et al. 1990) and study the axis ratio - environment relationship for elliptical galaxies. They arrive at the conclusion that "local density is not an important factor in determining the flattening of ellipticals". The lack of redshift information constrains Lambas et al. (1992) to estimate the local density in projection. This dilutes the signal by inevitably counting galaxies that have similar celestial coordinates as neighbors, while in reality they are separated by a large distance in space. Furthermore, they use a fixed angular scale on the sky within which they count the number of neighbors. For nearby galaxies, this scale corresponds to a larger physical distance than for distant galaxies. Our plots (Figures 5 through 7 ) show that trends tend to get washed out as cylinder size increases. They are effectively mixing different cylinder sizes and in the process washing out any trend. Within this angular area, Lambas et al. (1992) label their galaxies as 'high-density' if there are more than two neighboring galaxies, and 'low-density' if there are two or less. This cut divides their sample into an approximately equal number of high and low density galaxies. They claim that there is no statistical difference in the observed distribution of their high and low density ellipticals. We performed a Komolgorov-Smirnov test on the distribution of axis ratios derived from the moments, 
for the de galaxies with $M_{r} \in[-20,-22]$ in $0.5 h^{-1}$ Mpc cylinders. The KS probability is minimized when we place the cut between low and high densities at eight nearest neighbors; this results in a probability of $P_{K S}=7 \times 10^{-8}$. Instead of dividing our sample into two equal parts, this cut corresponds to a low/high density split of $\sim 9 / 1$. Thus Lambas et al. (1992) did not see the trend for two reasons: density in projection is a poor measure, and the difference in shape is most significant in very high density environments.

One open problem is whether or not field ellipticals are created and evolve in a fundamentally different way from those found in high density environments. Thomas et al. (2005) have shown that the massive early type galaxies in low density environments appear to have younger stellar populations and be slightly more metal rich than the ones in high density environments. Ryden, Forbes, \& Terlevich (2001) have shown that elliptical galaxies with younger stellar populations tend to be flatter in their inner regions than ones with older stellar populations. One possible explanation for such behavior is that the central black hole in older ellipticals has had a longer time to randomize the orbits of stars near the center. In light of our result that the inner regions of $d e$ galaxies tend to be more flattened in low density versus high density regions, these two previous studies may point to a more fundamental cause for our observed trends. However, using an age environment correlation to explain our results is no more fundamental than simply saying that axis ratio and environment are correlated. Instead, the missing link is in understanding what processes form centrally concentrated galaxies such as massive ellipticals.

Bournaud et al. (2005) have used numerical simulations to show that it is possible to form elliptical systems from the merger of a large disk with multiple smaller disks. When a large disk galaxy is merged with one or two small disks (with the mass ratio of the large disk to the small disk being 1:7) it is found that a galaxy of an intermediate type is created. Such a galaxy may correspond to the $e x / d e$ and de/ex galaxies observed in the SDSS. A well known fact is that the merger of two spirals of roughly equal mass leads to the creation of an elliptical (e.g. Barnes (1992); Hernandez et al. (2004); Bournaud et al. (2005)). However, it has not been shown that the most common way of creating an elliptical is through the merger of two spirals. Other numerical studies (Hernandez et al. 2004) claim to find evidence that there exist elliptical galaxies that appear not to have been formed by a major merger of spirals. Such studies show that different merger histories can lead to the creation of a galaxy with a de Vaucouleurs profile. Moreover, different merger histories lead to galaxies with different intrinsic axis ratios (Barnes 1992). If it was determined conclusively that certain types of mergers are favored in high versus low density regions, it would be possible to deduce the average axis ratio of galaxies in each environment. Conversely, if different merger histories produce characteristically different axis ratios, it may be possible to work backwards and deduce which merger histories are more common in a given environment. In any case, the axis ratio of a galaxy, in its outer regions, could also depend on harassment from neighboring galaxies. However, without knowing which merger histories are favored in different environments, and assuming that the observed environment is similar to the pre-merger environment, it is not possible to determine the 'initial' axis ratio of a galaxy and thus separate the the effect of harassment from that of the 
merger history.

One intriguing fact uncovered is that the $e x$ and $e x / d e$ galaxies with $M_{r} \sim-20$ are flatter, on average, in denser environments. These galaxies are primarily flattened disk galaxies, with a past free from major mergers. In a high density environment, a galaxy may be harassed by numerous encounters in the past, or may have a tidal tail from a recent close encounter. These processes may distort the apparent shape of galaxies in dense regions. However, we visually inspected all the $e x$ and $e x / d e$ galaxies that have 25 or more neighboring galaxies in a cylinder of radius $0.5 h^{-1}$ Mpc. There were 35 such galaxies, of which only one appeared to have a tidal tail and only one was a possible recent merger remnant. We also inspected $35 \mathrm{ex}$ and $e x / d e$ galaxies with at most one neighbor; none of them had obvious tidal tails or asymmetric distortions. Almost all galaxies in our visual samples were separated from their neighbors by a distance significantly larger than the galaxy's visual radius. If the greater average flattening at higher density is due to tidal effects, then the tidal distortions must be subtle, rather than taking the form of strong tidal tails.

The SDSS has recently been used to study the correlation of many galactic properties with each other. Exhaustive studies, such as those produced by Blanton et al. (2003a), Hogg et al. (2003) and Hogg et al. (2004), arrive at the conclusion that luminosity and color are the fundamental galactic properties from which all correlations of other properties with environment are derived. Such claims are tested in and are in reasonable agreement with numerical simulations as described, for example, in Berlind et al. (2004). However, we find a trend, namely the apparent axis ratio local galaxy density correlation, for galaxies of the same $r$ band luminosity and surface brightness profile type, that is not due to a correlation of luminosity or color with environment.

We thank Andreas Berlind and the anonymous referee for useful discussion and feedback.

Funding for the creation and distribution of the SDSS Archive has been provided by the Alfred P. Sloan Foundation, the Participating Institutions, the National Aeronautics and Space Administration, the National Science Foundation, the U.S. Department of Energy, the Japanese Monbukagakusho, and the Max Planck Society. The SDSS website is http://www.sdss.org/. The SDSS is managed by the Astrophysical Research Consortium (ARC) for the Participating Institutions. The Participating Institutions are The University of Chicago, Fermilab, the Institute for Advanced Study, the Japan Participation Group, The Johns Hopkins University, Los Alamos National Laboratory, the Max-Planck-Institute for Astronomy (MPIA), the Max-Planck-Institute for Astrophysics (MPA), New Mexico State University, University of Pittsburgh, Princeton University, the United States Naval Observatory, and the University of Washington. 


\section{REFERENCES}

Abadi, M. G., Navarro, J. F., Steinmetz, M., \& Eke, V. R. 2003, ApJ, 591, 499

Abazajian, K., et al. 2003, AJ, 126, 2081

Abazajian, K. et al. 2004, AJ, 128, 502

Abazajian, K. et al. 2005, AJ, submitted (astro-ph/0410239)

Adelman-McCarthy, J. K. et al. 2005 astro-ph/0507711

Barnes, J. E. 1992, ApJ, 393, 484

Bell, E. et al., 2005, astro-ph/0506425

Berlind, A. A., et al. 2004, astro-ph/0406633

Bernstein, G. M., \& Jarvis, M. 2002, AJ, 123, 583

Bertschinger, E. 1998, ARAA, 36, 599

Binney, J., de Vaucouleurs, G. 1981, MNRAS, 194, 679

Blanton, M. R., Eisenstein, D., Hogg, D. W., Zehavi, I. 2004, astro-ph/0411037

Blanton, M. R., et al. 2003a, ApJ, 594, 186

Blanton, M. R., Lin, H., Lupton, R., Maley, F. M., Young, N., Zehavi, I., \& Loveday, J. 2003b, AJ, 125. 2276

Bournaud, F., Jog, C. J., \& Combes, F. 2005, astro-ph/0503189

de Vaucouleurs, G. 1948, Ann. d'Astrophys. 11, 247

de Vaucouleurs, G. 1959, Handb. Phys. 53, 275

Dressler, A., 1980, ApJ, 236, 351

Elmegreen, D. M., Elmegreen, B. G., Hirst, A. C. 2004, ApJ, 604, L21

Freeman, K. C. 1970, ApJ, 160, 811

Fukugita, M., Ichikawa, T., Gunn, J. E., Doi, M., Shimasaku, K., \& Schneider, D. P. 1996, AJ, 111,1748

Goto, T., 2003, MNRAS, 346, 601

Gunn, J. E., et al. 1998, AJ, 116, 3040 
Hernandez, X., \& Lee, H. W. 2004, MNRAS, 347, 1304

Hirata, C., \& Seljak, U. 2003, MNRAS, 343, 459

Hogg, D. W., et al. 2003, ApJ, 585, L5

Hogg, D. W., et al. 2004, ApJ, 601, L29

Hubble, E. 1926, ApJ, 64, 321

Hubble, E., \& Humason, M. 1931, ApJ, 74, 43

Lambas, D. G., Maddox, S. J., \& Loveday, J. 1992, MNRAS, 258, 404

Maddox, S. J., Sutherland, W. J., Efstathiou, G., Loveday, J. 1990, MNRAS, 243, 692

Naim, A., et al. 1995, MNRAS, 274, 1107

Nuijten, M. J. H. M., Simard, L., Gwyn, S., Röttgering, H., J., A. 2005, ApJ, 626, L77

Postman, M., et al. 2005, AJ, 623, 721

Roberts, M. S., \& Haynes, M. P. 1994, ARA\&A, 32, 115

Robertson, B., Hernquist, L., Bullock, J.S., Cox, T.J., Di Matteo, T., Springel, V., Yoshida, N. 2005, astro-ph/0503369

Ryden, B. S., Forbes, D. A., \& Terlevich, A. I. 2001, MNRAS, 326, 1141

Sandage, A., Freeman, K. C., \& Stokes, N. R. 1970 ApJ, 160, 831

Sérsic, J. L. 1968, Atlas de Galaxias Australes (Cordoba: Obs. Astron.)

Sheldon, E. S., et al. 2004, AJ, 127, 2544

Smith, J. A., et al. 2002, AJ, 123, 2121

Stoughton, C., et al. 2002, AJ, 123, 485

Tanaka, M., Goto, T., Okamura, S., Shimasaku, K., \& Brinkmann, J. 2004, AJ, 128, 2677

Thomas, D., Maraston, C., Bender, R., \& De Oliveira, C. M. 2005, ApJ, 621, 673

Vincent, R. A., \& Ryden, B. S. 2005, ApJ, 623, 137

York, D. G. et al. 2000, AJ, 120, 1579 


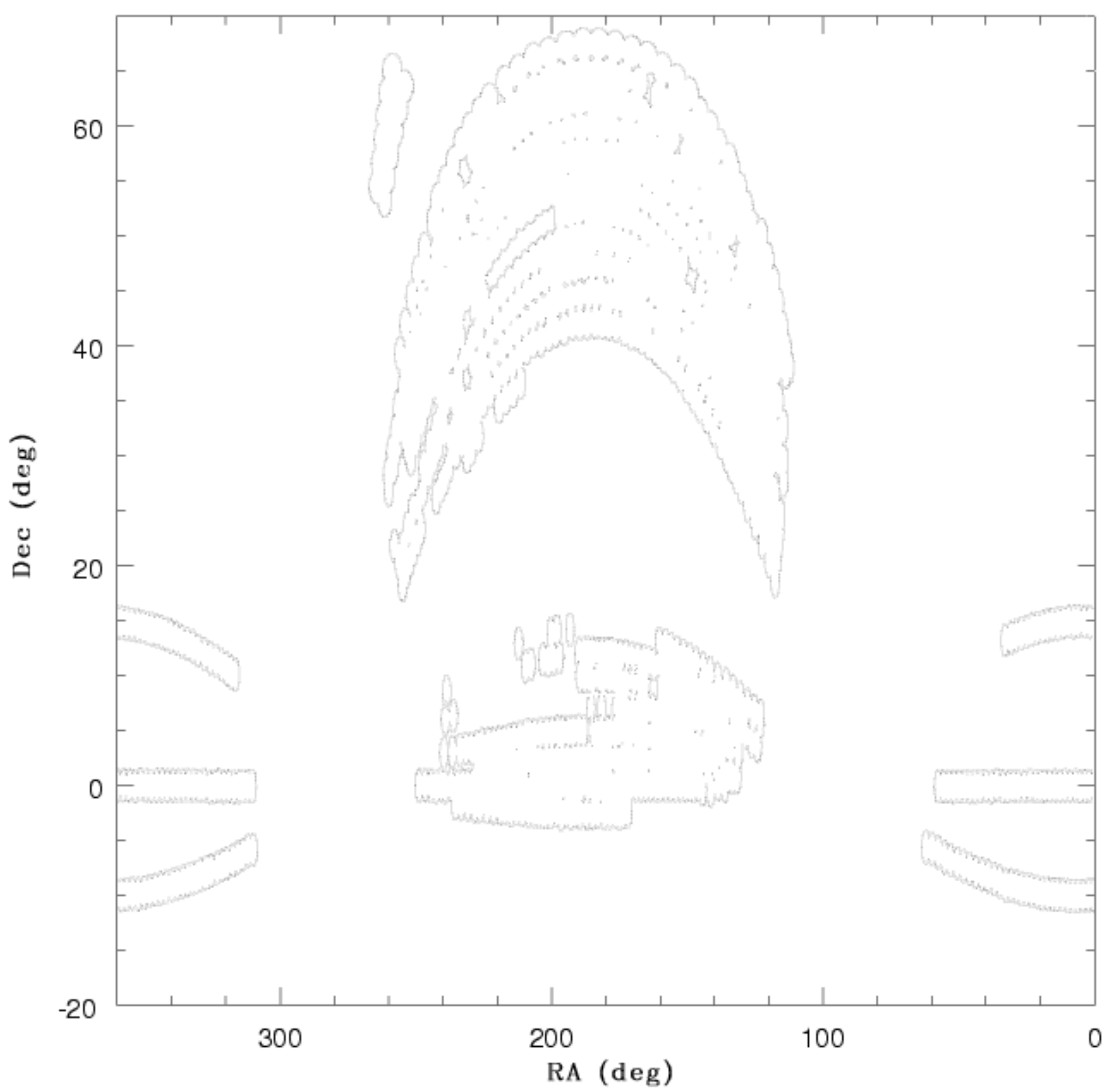

Fig. 1.- Boundary of the SDSS DR4 spectroscopic survey. 


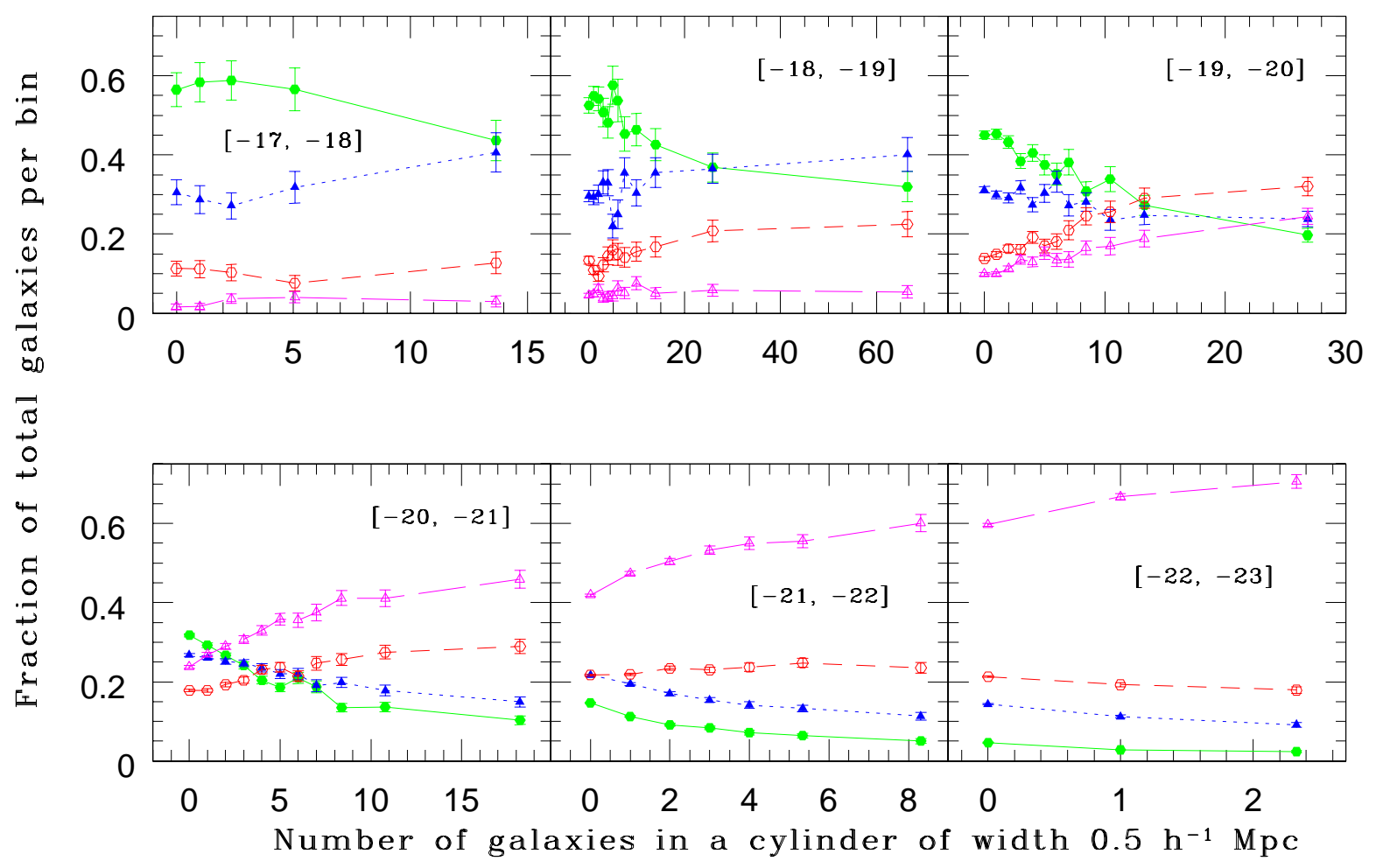

Fig. 2.- Profile type - environment relation, using cylinders of width $0.5 h^{-1} \mathrm{Mpc}$. Panels display the fraction of each surface brightness profile type vs. the number of local galaxies for the magnitude ranges $[-17,-18],[-18,-19],[-19,-20],[-20,-21],[-21,-22]$, and $[-22,23]$. Solid, green lines with filled circles indicate $e x$ type galaxies; dotted, blue lines with filled triangles are for $e x / d e$ type galaxies; short dashed, red lines with open circles are for de/ex type galaxies; long dashed, magenta lines with open triangle are for de type galaxies.

Table 1. Galaxy Profile Types: Binned by Luminosity

\begin{tabular}{rrrrrrrrr}
\hline \hline \multirow{2}{*}{$\begin{array}{c}\text { Luminosity } \\
\text { Bin Width }\end{array}$} & \multirow{2}{*}{$\begin{array}{c}\text { Total } \\
\text { Galaxies }\end{array}$} & \multicolumn{3}{c}{ Fraction of each profile type } & \multicolumn{3}{c}{ Cylinder width } \\
& & ex/de & $d e / e x$ & $d e$ & $0.5 h^{-1} \mathrm{Mpc}$ & $2 h^{-1} \mathrm{Mpc}$ & $8 h^{-1} \mathrm{Mpc}$ \\
\hline$-17 \rightarrow-18$ & 1610 & 0.56 & 0.30 & 0.11 & 0.03 & 1140 & 253 & $\ldots$ \\
$-18 \rightarrow-19$ & 5915 & 0.50 & 0.31 & 0.14 & 0.05 & 5267 & 2410 & $\ldots$ \\
$-19 \rightarrow-20$ & 15580 & 0.41 & 0.30 & 0.17 & 0.12 & 14698 & 9192 & $\ldots$ \\
$-20 \rightarrow-21$ & 46755 & 0.27 & 0.25 & 0.20 & 0.28 & 45549 & 36208 & 8856 \\
$-21 \rightarrow-22$ & 81037 & 0.12 & 0.20 & 0.22 & 0.46 & 79988 & 70560 & 26975 \\
$-22 \rightarrow-23$ & 57900 & 0.04 & 0.14 & 0.21 & 0.61 & 57482 & 54219 & 30614 \\
\hline
\end{tabular}




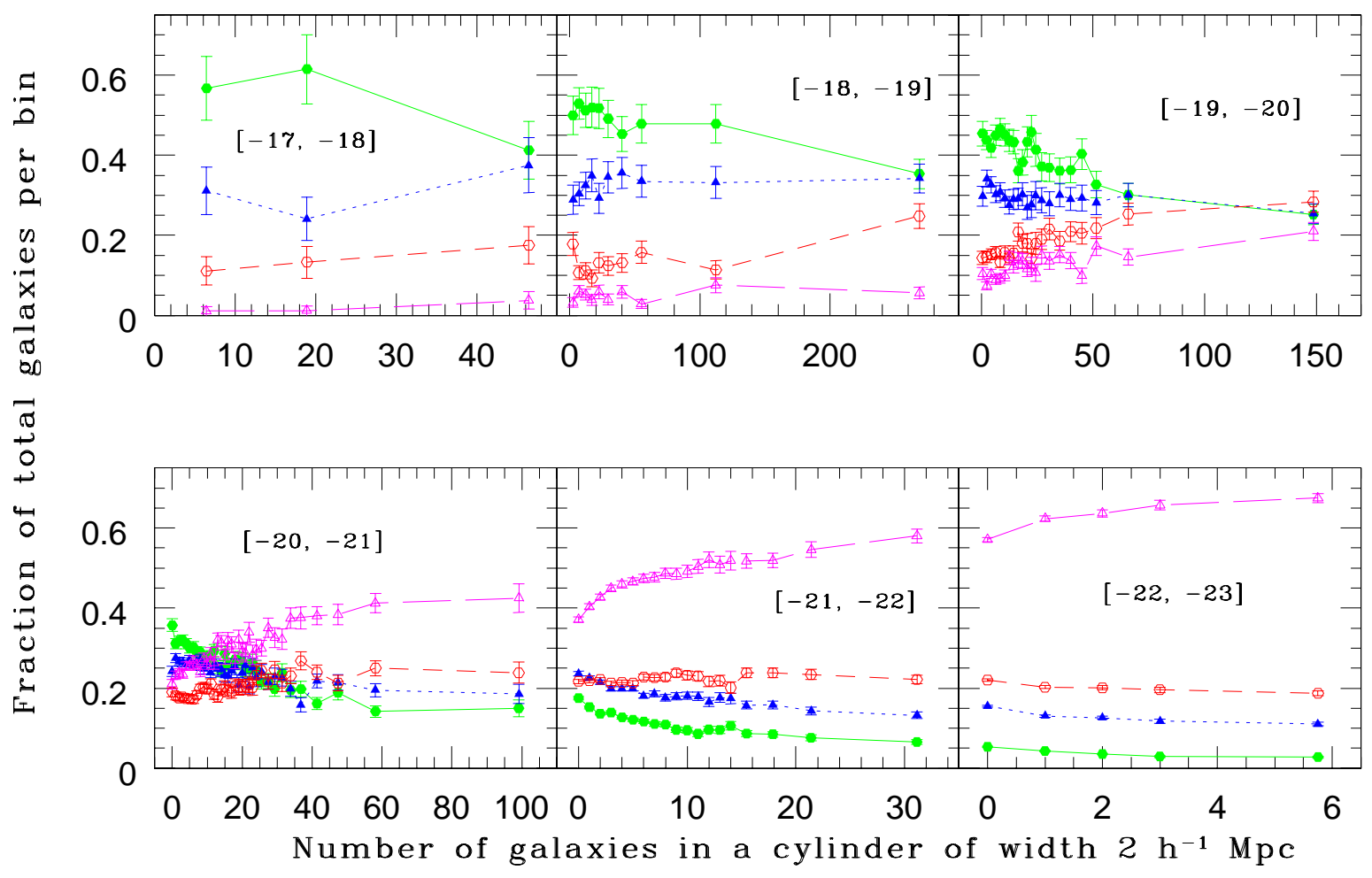

Fig. 3. - Same as Figure 2, but with cylinders of width $2 h^{-1}$ Mpc.

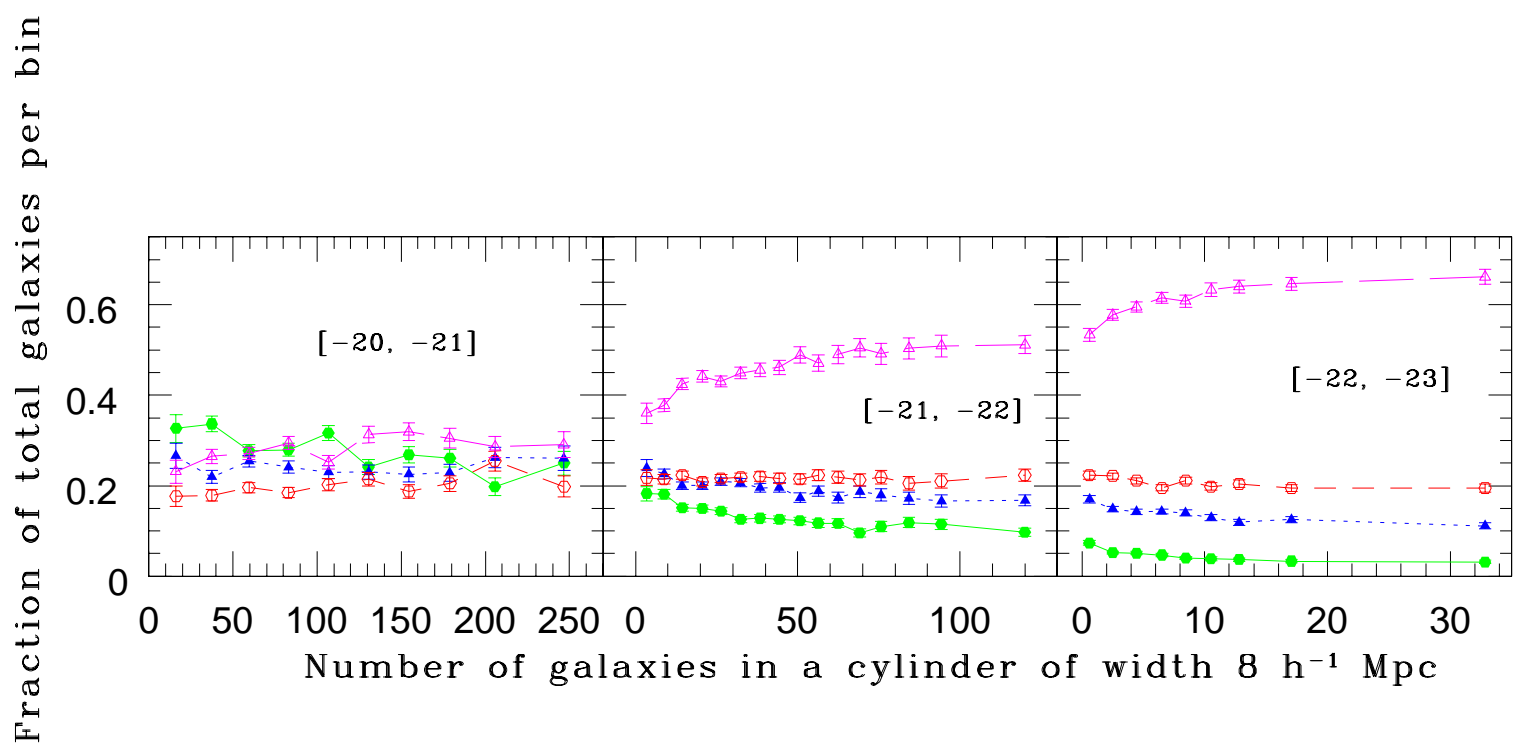

Fig. 4. - Same as Figure 3, but with cylinders of width $8 h^{-1} \mathrm{Mpc}$. 

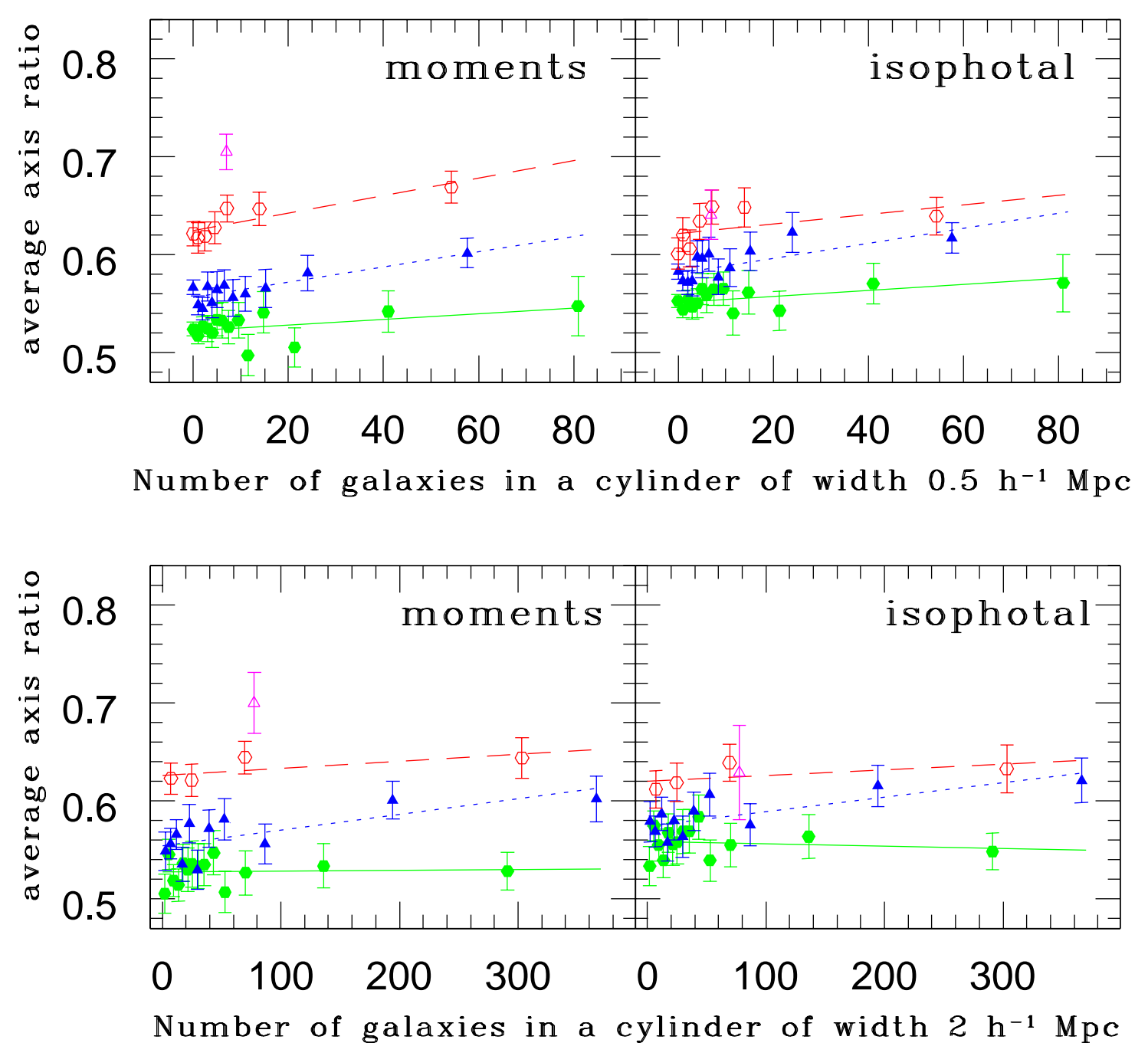

Fig. 5.- Apparent axis ratio - environment relation, using target galaxies in the absolute magnitude range $M_{r} \in[-18,-20]$ and cylinders of width $0.5 h^{-1} \mathrm{Mpc}$ (top panel) and $2 h^{-1} \mathrm{Mpc}$ (bottom panel). Straight lines are $\chi^{2}$ fits to the binned data. Left panel plots are for the axis ratio as derived by the moments of the light distribution; right panel plots are for the axis ratio derived from the $25 \mathrm{mag} \operatorname{arcsec}^{-2}$ isophote. The line types, colors, and point types are identical to those in figure 2 . 


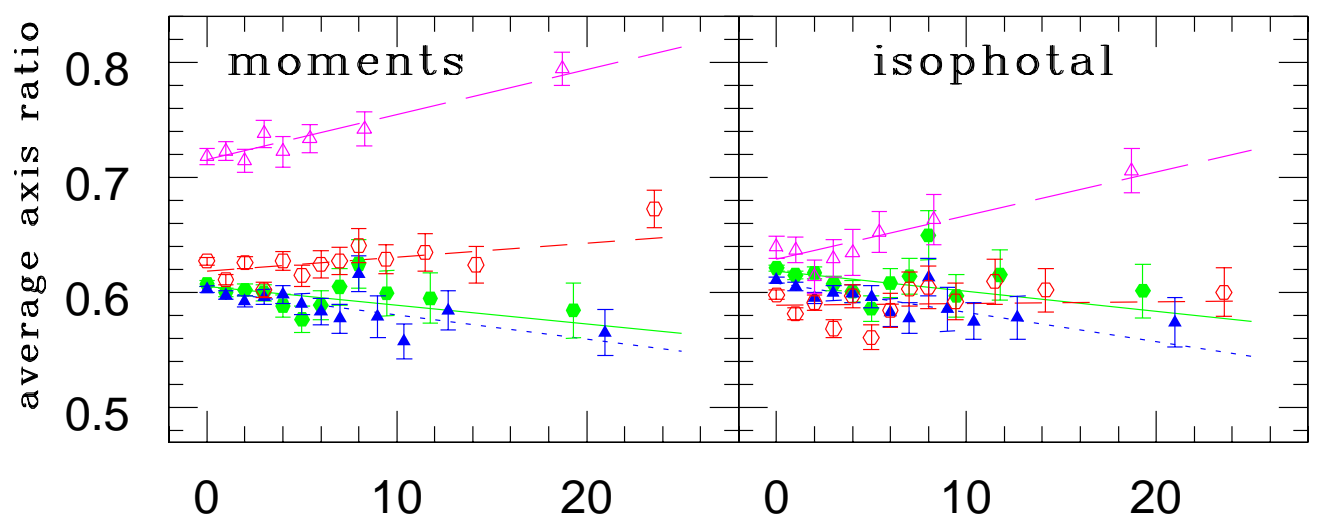

Number of galaxies in a cylinder of width $0.5 \mathrm{~h}^{-1} \mathrm{Mpc}$

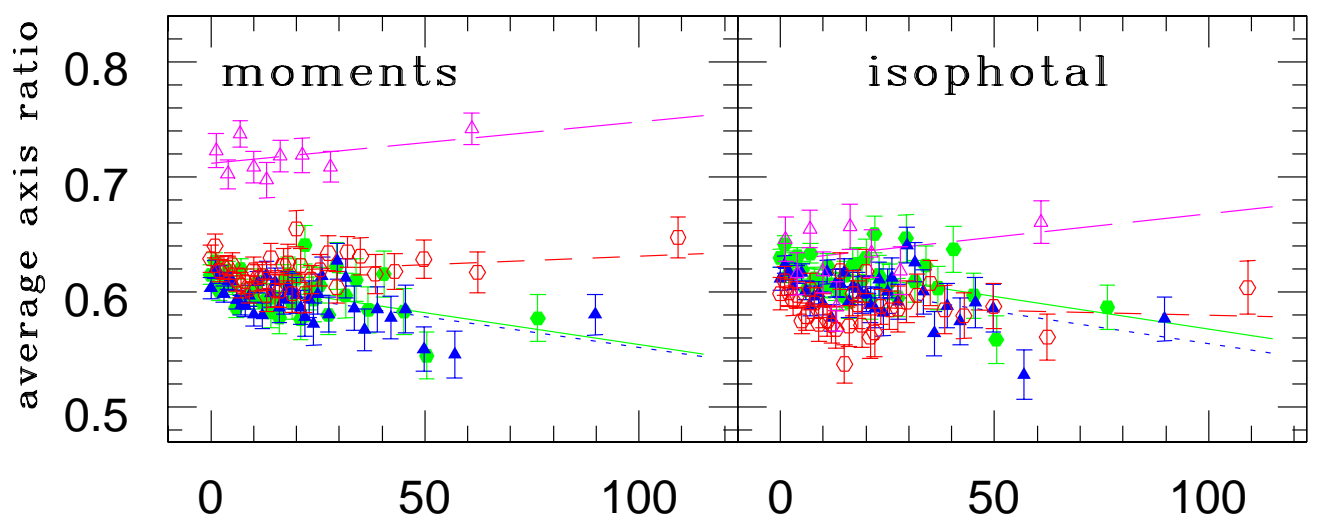

Number of galaxies in a cylinder of width $2 \mathrm{~h}^{-1} \mathrm{Mpc}$

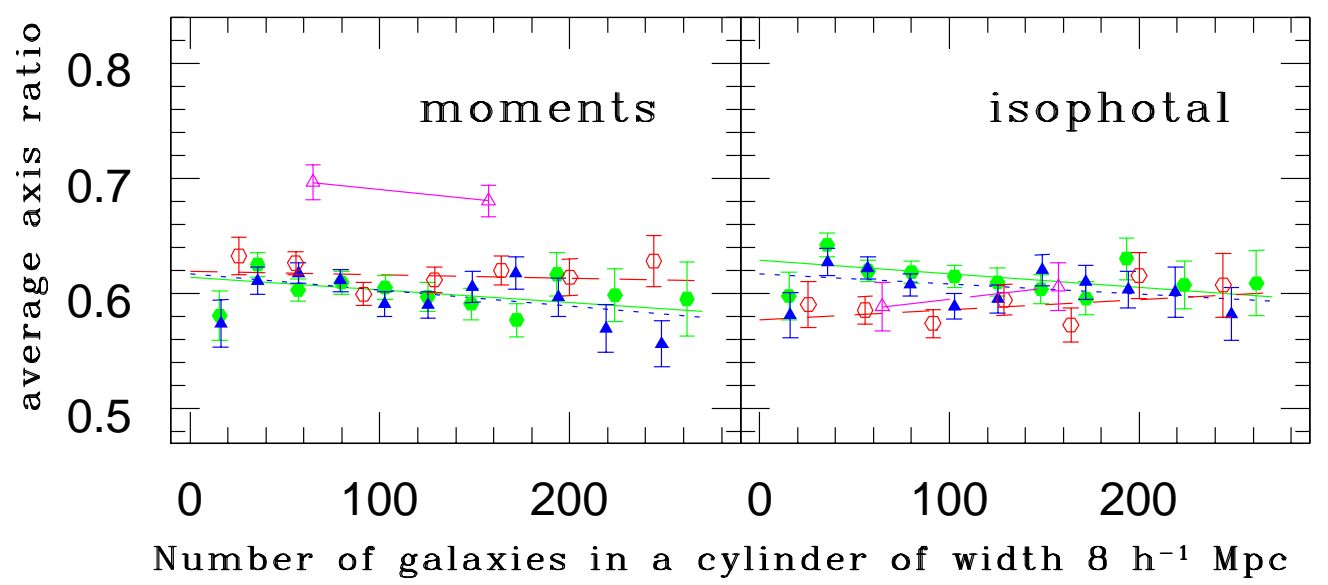

Fig. 6. - Same as Figure 5, but with target galaxies in the absolute magnitude range $M_{r} \in$ $[-20,-22]$ and cylinders of width $0.5 h^{-1} \mathrm{Mpc}$ (top panel), $2 h^{-1} \mathrm{Mpc}$ (middle panel) and $8 h^{-1}$ Mpc (bottom panel). 

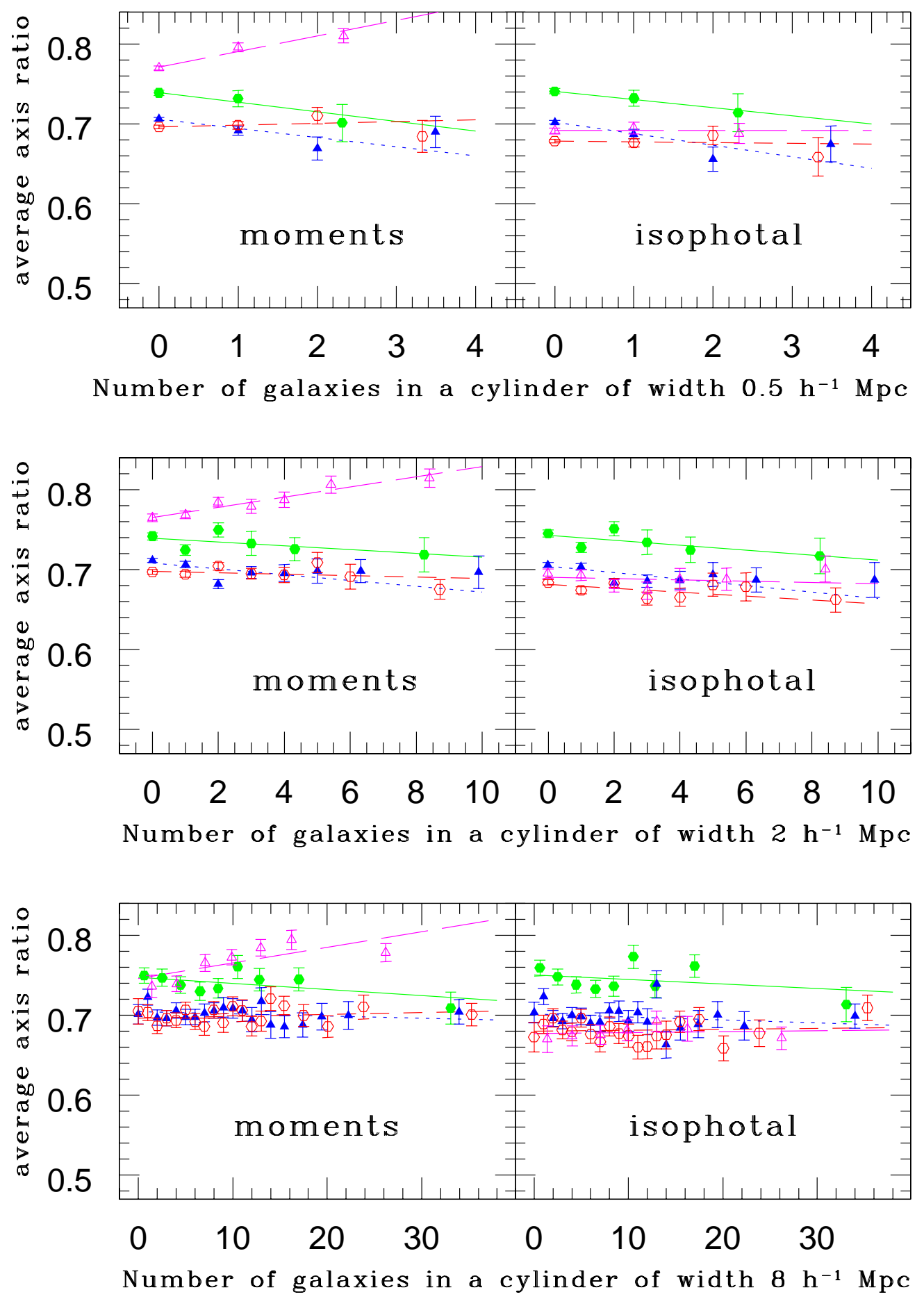

Fig. 7.- Same as Figure 5, but with target galaxies in the absolute magnitude range magnitude range $M_{r} \in[-20,-22]$ and cylinders of width $0.5 h^{-1} \mathrm{Mpc}$ (top panel), $2 h^{-1} \mathrm{Mpc}$ (middle panel) and $8 h^{-1} \mathrm{Mpc}$ (bottom panel). 
Table 2. Galaxy Axis Ratio: Binned by Luminosity

\begin{tabular}{|c|c|c|c|c|c|c|c|c|}
\hline \multirow{2}{*}{$\begin{array}{l}\text { Luminosity } \\
\text { Bin Width }\end{array}$} & \multirow{2}{*}{$\begin{array}{c}\text { Total } \\
\text { Galaxies }\end{array}$} & \multicolumn{4}{|c|}{ Fraction of each profile type } & \multicolumn{3}{|c|}{ Cylinder width } \\
\hline & & $e x$ & $e x / d e$ & $d e / e x$ & $d e$ & $0.5 h^{-1} \mathrm{Mpc}$ & $2 h^{-1} \mathrm{Mpc}$ & $8 h^{-1} \mathrm{Mpc}$ \\
\hline$-18 \rightarrow-20$ & 8669 & 0.53 & 0.35 & 0.11 & 0.01 & 7691 & 3459 & $\ldots$ \\
\hline$-20 \rightarrow-22$ & 39964 & 0.37 & 0.39 & 0.20 & 0.04 & 38901 & 30536 & 7180 \\
\hline$-22 \rightarrow-\infty$ & 20211 & 0.12 & 0.37 & 0.37 & 0.14 & 20055 & 18731 & 10068 \\
\hline
\end{tabular}

Table 3. Galaxy Axis Ratio: Galaxies with $M_{r} \in[-18,-20]$

\begin{tabular}{cccccccc}
\hline \hline \multirow{2}{*}{$\begin{array}{c}\text { Profile } \\
\text { Type }\end{array}$} & \multirow{2}{*}{$\begin{array}{c}\text { Width } \\
\left(h^{-1} \mathrm{Mpc}\right)\end{array}$} & \multicolumn{3}{c}{ Isophotal } & \multicolumn{4}{c}{ Moments } \\
\hline$\chi^{2} / \mathrm{DOF}$ & trend & $\sigma$ & $\chi^{2} / \mathrm{DOF}$ & trend & $\sigma$ \\
\hline$e x$ & 0.5 & 0.28 & + & 1.1 & 0.38 & + & 0.96 \\
$e x / d e$ & 0.5 & 0.50 & + & 2.8 & 0.43 & + & 2.9 \\
$d e / e x$ & 0.5 & 0.82 & + & 1.2 & 0.35 & + & 2.7 \\
\hline$e x$ & 2 & 0.49 & - & 0.36 & 0.41 & + & 0.11 \\
$e x / d e$ & 2 & 0.40 & + & 2.4 & 0.67 & + & 2.6 \\
$d e / e x$ & 2 & 0.22 & + & 0.58 & 0.22 & + & 0.86 \\
\hline
\end{tabular}

Table 4. Galaxy Axis Ratio: Galaxies with $M_{r} \in[-20,-22]$

\begin{tabular}{cccccccc}
\hline \hline \multirow{2}{*}{$\begin{array}{c}\text { Profile } \\
\text { Type }\end{array}$} & \multirow{2}{*}{$\left.h^{-1} \mathrm{Mpc}\right)$} & \multicolumn{3}{c}{ Isophotal } & \multicolumn{4}{c}{ Moments } \\
$\chi^{2} / \mathrm{DOF}$ & trend & $\sigma$ & $\chi^{2} / \mathrm{DOF}$ & trend & $\sigma$ \\
\hline$e x$ & 0.5 & 1.2 & - & 2.5 & 0.83 & - & 2.2 \\
$e x / d e$ & 0.5 & 0.66 & - & 4.2 & 0.74 & - & 3.7 \\
$d e / e x$ & 0.5 & 1.9 & + & 0.23 & 2.0 & + & 2.4 \\
$d e$ & 0.5 & 0.59 & + & 3.6 & 0.34 & + & 4.9 \\
\hline$e x$ & 2 & 1.4 & - & 3.6 & 1.4 & - & 3.4 \\
$e x / d e$ & 2 & 1.3 & - & 4.0 & 1.2 & - & 4.0 \\
$d e / e x$ & 2 & 1.1 & - & 0.52 & 0.93 & + & 1.2 \\
$d e$ & 2 & 1.3 & + & 1.1 & 0.93 & + & 1.4 \\
\hline$e x$ & 8 & 0.75 & - & 1.7 & 0.76 & - & 1.6 \\
$e x / d e$ & 8 & 1.0 & - & 1.3 & 1.2 & - & 2.1 \\
$d e / e x$ & 8 & 0.61 & + & 0.88 & 0.77 & - & 0.37 \\
\hline & & & & & & &
\end{tabular}


Table 5. Galaxy Axis Ratio: Galaxies with $M \in[-22,-\infty]$

\begin{tabular}{|c|c|c|c|c|c|c|c|}
\hline \multirow{2}{*}{$\begin{array}{l}\text { Profile } \\
\text { Type }\end{array}$} & \multirow{2}{*}{$\begin{array}{c}\text { Width } \\
\left(h^{-1} \mathrm{Mpc}\right)\end{array}$} & \multirow{2}{*}{\multicolumn{2}{|c|}{$\chi^{2} / \mathrm{DOF} \quad \begin{array}{c}\text { Isophotal } \\
\text { trend }\end{array}$}} & \multirow[b]{2}{*}{$\sigma$} & \multicolumn{3}{|c|}{ Moments } \\
\hline & & & & & $\chi^{2} / \mathrm{DOF}$ & trend & $\sigma$ \\
\hline$e x$ & 0.5 & 0.012 & - & 1.3 & 0.13 & - & 1.6 \\
\hline$e x / d e$ & 0.5 & 0.60 & - & 3.6 & 0.66 & - & 3.1 \\
\hline$d e / e x$ & 0.5 & 0.29 & + & 0.23 & 0.45 & + & 0.67 \\
\hline$d e$ & 0.5 & 0.0010 & + & $6.0 \times 10^{-4}$ & 0.55 & + & 5.5 \\
\hline$e x$ & 2 & 1.0 & - & 1.4 & 0.99 & - & 0.96 \\
\hline$e x / d e$ & 2 & 0.75 & - & 3.5 & 1.3 & - & 3.3 \\
\hline$d e / e x$ & 2 & 0.86 & - & 2.1 & 0.65 & - & 0.83 \\
\hline$d e$ & 2 & 0.35 & - & 0.86 & 0.45 & + & 5.0 \\
\hline$e x$ & 8 & 1.0 & - & 1.1 & 0.57 & - & 1.1 \\
\hline$e x / d e$ & 8 & 0.91 & - & 1.5 & 0.56 & - & 1.2 \\
\hline$d e / e x$ & 8 & 1.2 & + & 0.40 & 0.85 & + & 0.25 \\
\hline$d e$ & 8 & 0.38 & + & 0.35 & 1.8 & + & 2.4 \\
\hline
\end{tabular}

